\title{
LncRNA FEZF1-AS1 promotes non-small lung cancer cell migration and invasion through the up-regulation of NOTCH1 by serving as a sponge of miR-34a
}

Shangxiao Huang ${ }^{1 *}$, Chunjun $\mathrm{Li}^{2}$, Jianfeng Huang ${ }^{1}$, Penghui Luo ${ }^{1}$, Dunchang Mo ${ }^{1}$ and Hanlei Wang ${ }^{1}$

\begin{abstract}
Background: The involvement of IncRNA FEZF1-AS1 has been analyzed in many types of cancers, while its roles in non-small cell lung cancer (NSCLC) remains unclear. We then explored the role of FEZF1-AS1 in NSCLC.

Methods: qPCR and western blot were performed to measure gene expression. FEZF1-AS1, miR-34a, and NOTCH-1 were overexpressed to analyze the relationship between them. Transwell assays were performed to analyze the effects of transfections on cell invasion and migration.

Results: FEZF1-AS1 was up-regulated in NSCLC patients. Increased expression levels of FEZF1-AS1 were observed with the increase in clinical stages. Bioinformatics analysis showed that miR-34a can bind with FEZF1-AS1. In NSCLC tissues, NOTCH-1 and FEZF1-AS1 were positively correlated. In NSCLC cells, over-expression of FEZF1-AS1 resulted in up-regulated expressions of NOTCH-1, while miR-34a over-expression mediated down-regulated expressions of NOTCH-1. In addition, FEZF1-AS1 and miR-34a did not alter each other, while bioinformatics analysis showed that miR-34a can bind FEZF1-AS1. Analysis of cell migration and invasion showed increased cell invasion and migration rates after FEZF1-AS1 and NOTCH-1 over-expression. MiR-34a played the opposite role and reduced the effects of FEZF1-AS1 over-expression.
\end{abstract}

Conclusions: FEZF1-AS1 promoted NSCLC cell migration and invasion through the up-regulation of NOTCH1 by serving as a sponge of miR-34a.

Keywords: FEZF1-AS1, Non-small cell lung cancer, miR-34a, NOTCH-1

\section{Background}

For decades, lung cancer ranks the first place for both mortality and incidence among all cancers [1]. In 2018, $2,093,876$ new cases of lung cancer were diagnosed and $1,761,007$ deaths caused by lung cancer were recorded $[1,2]$. NSCLC accounts for more than $85 \%$ of all cases

\footnotetext{
* Correspondence: fmlrpweubnen81@163.com

'Department of Radiotherapy, The Third Affiliated Hospital of Guangxi Medical University, No.13 Dancun Road, Nanning, Guangxi 530031, People's Republic of China

Full list of author information is available at the end of the article
}

$[3,4]$. Moreover, most NSCLC patients are diagnosed with tumor metastasis to other parts of the body and the 5 -year overall survival rate is as low as $10 \%[5,6]$. Therefore, novel therapeutic regimens are needed.

Smoking habit is closely correlated with the incidence of NSCLC [7], while NSCLC also affects non-smokers [8]. As another factor, genetic alterations also contribute to the occurrence and progression of NSCLC [9]. Notch homolog 1 (NOTCH-1) plays critical roles in cancer development by inducing epithelial-mesenchymal transition, promoting cell stemness and enhancing cell

(c) The Author(s). 2020 Open Access This article is licensed under a Creative Commons Attribution 4.0 International License, which permits use, sharing, adaptation, distribution and reproduction in any medium or format, as long as you give appropriate credit to the original author(s) and the source, provide a link to the Creative Commons licence, and indicate if changes were made. The images or other third party material in this article are included in the article's Creative Commons licence, unless indicated otherwise in a credit line to the material. If material is not included in the article's Creative Commons licence and your intended use is not permitted by statutory regulation or exceeds the permitted use, you will need to obtain permission directly from the copyright holder. To view a copy of this licence, visit http://creativecommons.org/licenses/by/4.0/. The Creative Commons Public Domain Dedication waiver (http://creativecommons.org/publicdomain/zero/1.0/) applies to the data made available in this article, unless otherwise stated in a credit line to the data. 
invasion $[10,11]$. Some miRNAs, such as miR-34a, targets NOTCH-1 to inhibit cancer progression [12]. Through different ways, such as miRNA sponge or methylation regulator, lncRNAs interact with miRNAs to participate in diverse biological processes [13]. LncRNA FEZF1-AS1 has been reported to promote the progression of several cancers, such as lung cancer [14, 15], while the functionality remains to be further elucidated. In this study, we performed RNA-RNA interaction prediction using IntaRNA 2.0 and we found that miR-34a may bind FEZF1-AS1. Therefore, we will be focused to analyze the relationship between FEZF1-AS1 and miR-34a in NSCLC and the effects on NOTCH-1.

\section{Methods}

\section{NSCLC patients}

In this study, we selected 39 male and 27 female NSCLC patients (age: 35 to 67 year-old; mean age: $51.1 \pm 5.6$ year-old) from 177 cases of NSCLC admitted to The Third Affiliated Hospital of Guangxi Medical University between March 2016 and April 2019. This study was approved The Third Affiliated Hospital of Guangxi Medical University Ethics Committee. Inclusion criteria: 1) newly diagnosed NSCLC; 2) no therapies were initiated. Exclusion criteria:1) complicated with other clinical disorders; 2) recurrent NSCLC; 3) therapies were initiated; 4) previous history and/or family history of malignancies. 66 NSCLC patients were informed with the experiment design and potential publication of this paper, and all 66 patients signed informed consent. Clinical staging was performed using AJCC system. Based on clinical data, the 66 patients included 10,12, 23 and 21 cases at stage I-IV, respectively.

\section{NSCLC tissue samples and cells}

Fine needle aspiration was performed on all patients to collect paired NSCNC and non-tumor specimens. Following histological confirmation, all tissue samples were immediately subjected to RNA isolations and following experiments.

H1993 human NSCLC cell line (ATCC, USA) was used in this study. Cell culture medium was prepared by mixing RPMI-1640 Medium (90\%) with FBS (10\%). A 95\% humidity and 5\% $\mathrm{CO}_{2}$ incubator was used to cultivate cells.

\section{Vectors, miRNA mimic and transfections}

Negative control (NC) miRNA and the mimic of miR34a, as well as vectors (pcDNA3.1) expressing NOTCH1 and FEZF1-AS1, were from GenePharma (Shanghai, China). Vector $(1 \mu \mathrm{g})$ or miRNA $(40 \mathrm{nM})$ was transfection into $4 \times 10^{6} \mathrm{H} 1993$ cells using lipofectamine 2000 (GenePharma). To perform NC experiments, H1993 cells were transfected with empty pcDNA3.1 vector or NC miRNA. To perform Control (C) experiment, cells without transfections were cultivated until the end of transfections. Cells were cultivated for further $48 \mathrm{~h}$ after transfections before use.

\section{RNA extractions}

H1993 cells were harvested and counted. All tissue samples (about $0.012 \mathrm{~g}$ ) were ground into powder in liquid nitrogen. Total RNAs in $4 \times 10^{5}$ cells or tissue samples were extracted using Ribozol (Sigma-Aldrich, USA). To harvest all types of RNAs (such as miRNAs), RNAs were precipitated and washed using $85 \%$ ethanol.

\section{RT-qPCR}

DNase I was used to incubate with RNA samples for 90 min at $37^{\circ} \mathrm{C}$ to achieve genomic DNA removal. In order to measure the expression levels of FEZF1-AS1 and NOTCH-1 mRNA, total RNAs were reverse transcribed into cDNA and qPCRs were performed with GAPDH as internal control. Expression of miR-34a was determined using All-in-One ${ }^{\text {тм }}$ miRNA qRT-PCR Detection Kit (Genecopoeia). U6 was included to sever as the
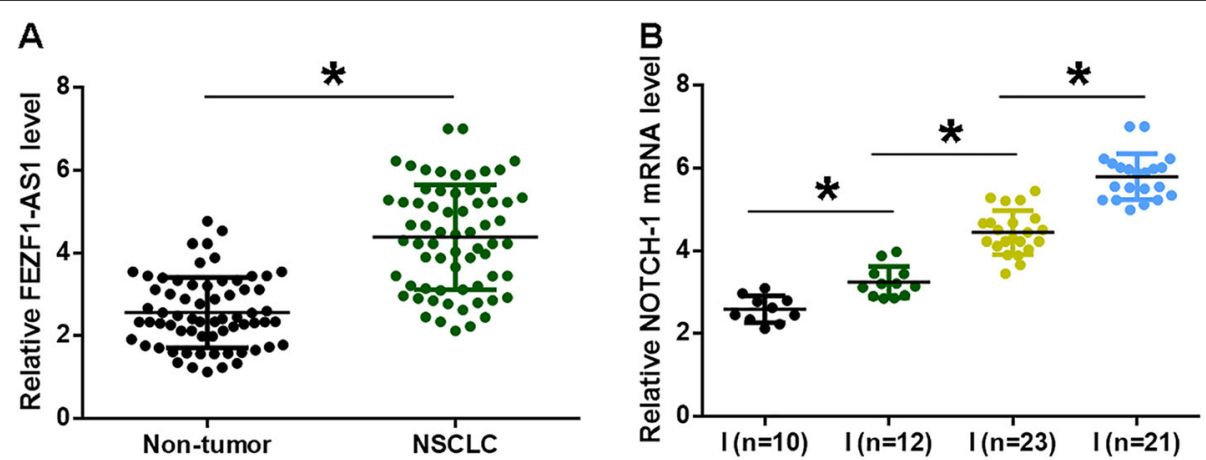

Fig. 1 FEZF1-AS1 was up-regulated with an increase in clinical stages of NSCLC patients. QPCR and paired t-test were used to measure and compare expression levels of FEZF1-AS1 between two types of tissues (NSCLC vs. non-tumor) (a). ANOVA (one-way) and Tukey test were used to compare expression levels of FEZF1-AS1 in NSCLC tissues among different clinical stages (b). Data of 3 replicates were presented, ${ }^{*}, p<0.05$ 
endogenous control of miR-34a. Ct values of three technical replicates were normalized using $2^{-\Delta \Delta C T}$ method.

\section{RNA interaction prediction}

The interaction between miR-34a and FEZF1-AS1 was predicted using an online program named IntaRNA 2.0 (http://rna.informatik.uni-freiburg.de/IntaRNA/Input.jsp). All parameters were the default. FEZF1-AS1 was set to be the long sequence and miR-34a was set to be the short sequence.

\section{Western blot}

At $24 \mathrm{~h}$ post-transfection, H1993 cells were counted and total proteins in $4 \times 10^{5}$ cells were extracted using RIPA solution (GenePharma). Total proteins were denatured in boiling water for $5 \mathrm{~min}$. Proteins were then separated by $10 \%$ SDS-PAGE gel, transferred to PVDF membranes, and PBS (5\% non-fat milk) was used to block membranes for $1 \mathrm{~h}$ at $24^{\circ} \mathrm{C}$. After that, rabbit anti-NOTCH-1 (1: 1200, ab52301, Abcam) and GAPDH (1: 1200, ab37168, Abcam) primary antibodies were used to incubate the membranes for $18 \mathrm{~h}$ at $4{ }^{\circ} \mathrm{C}$. After that, further incubation with IgG H\&L (IgG) (1:1000; ab6721; Abcam) secondary antibody was performed for $2 \mathrm{~h}$ at $24^{\circ} \mathrm{C}$. Signal development was performed by incubating membranes with RapidStep ${ }^{\text {tw }}$ ECL detection reagent (EMD Millipore) for $5 \mathrm{~min}$. Signals were processed using Image J v1.47 software.

\section{Transwell assays}

At $24 \mathrm{~h}$ post-transfection, $\mathrm{H} 1993$ cells were counted and transferred to upper Transwell chamber $\left(4 \times 10^{3}\right.$ cells in $0.1 \mathrm{ml}$ serum-free medium per well). Matrigel (Millipore)coated membranes were used in invasion assay and uncoated membranes were used in migration assay. In both cases, lower chamber was filled with medium supplemented $20 \%$ FBS. Under aforementioned conditions, cells were cultivated for $12 \mathrm{~h}$ and $0.5 \%$ crystal violet (Sigma-Aldrich) was used to stain cell for 20 min at room temperature. An optical microscope was used to observe stained cells.

\section{Data analysis}

Mean \pm SD values of 3 biological replicates were calculated. Paired $t$ test was used to compare paired tissues. ANOVA Tukey's test was used to compare multiple groups. Linear regression was used for correlation analysis. Patients were first grouped in to high and low $(n=33)$ FEZF1-AS1 level groups. The cutoff value was the median FEZF1-AS1 expression level in NSCLC. Correlations between expression levels of FEZF1-AS1 and patients' clinical data were analyzed by Chisquared test. $p<0.05$ was statistically significant.

\section{Results}

FEZF1-AS1 was up-regulated with an increase in clinical stages of NSCLC patients

Analysis of RT-qPCR data revealed significantly higher expression levels of FEZF1-AS1 in NSCLC tissues

Table 1 Correlations between expression levels of FEZF1-AS1 in NSCLC tissues and patients' clinical data

\begin{tabular}{|c|c|c|c|c|c|c|}
\hline Items & Groups & Cases & High & Low & $x^{2}$ & $p$ value \\
\hline \multirow[t]{2}{*}{ Age } & $>50$ (years) & 35 & 19 & 16 & 0.55 & 0.46 \\
\hline & $<=50$ (years) & 31 & 14 & 17 & & \\
\hline \multirow[t]{2}{*}{ Gender } & Male & 39 & 22 & 17 & 1.57 & 0.21 \\
\hline & Female & 27 & 11 & 16 & & \\
\hline \multirow[t]{3}{*}{ Subtypes } & Adenocarcinoma & 28 & 14 & 14 & 0.13 & 0.94 \\
\hline & Squamous cell carcinoma & 27 & 13 & 14 & & \\
\hline & Large cell carcinoma & 11 & 6 & 5 & & \\
\hline \multirow[t]{4}{*}{ Tumor grade } & I & 12 & 3 & 9 & 18.10 & 0.00042 \\
\hline & ॥ & 16 & 3 & 13 & & \\
\hline & III & 17 & 13 & 4 & & \\
\hline & IV & 21 & 14 & 7 & & \\
\hline \multirow[t]{4}{*}{ Tumor stage } & । & 10 & 2 & 8 & 0.92 & 0.02 \\
\hline & $\|$ & 12 & 3 & 9 & & \\
\hline & III & 23 & 15 & 8 & & \\
\hline & IV & 21 & 13 & 8 & & \\
\hline \multirow[t]{2}{*}{ Smoking } & Yes & 41 & 21 & 20 & 0.06 & 0.80 \\
\hline & No & 25 & 12 & 13 & & \\
\hline \multirow[t]{2}{*}{ Drinking } & Yes & 46 & 22 & 24 & 0.29 & 0.59 \\
\hline & No & 20 & 11 & 9 & & \\
\hline
\end{tabular}



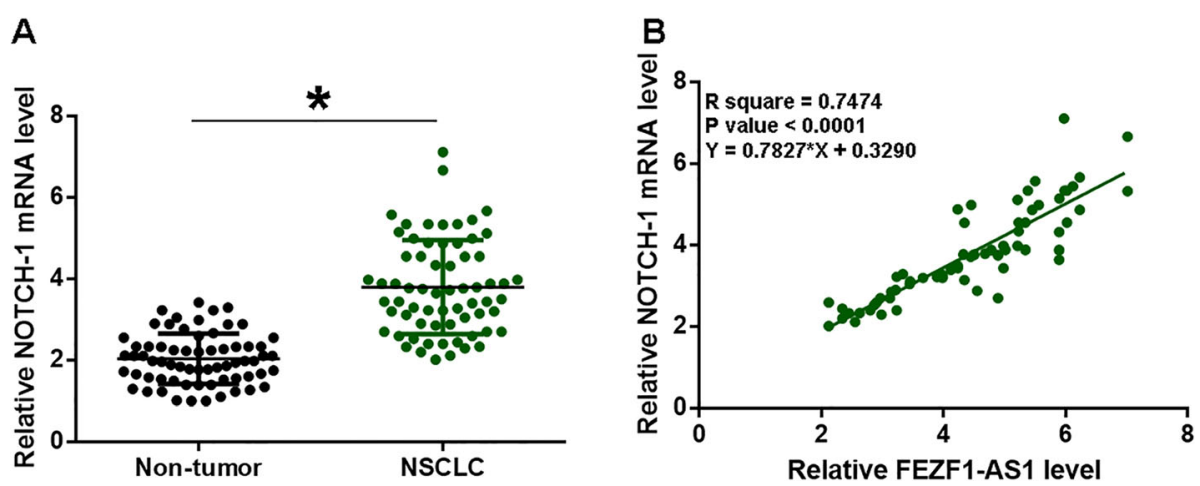

Fig. 2 NOTCH-1 mRNA was up-regulated and positively correlated with FEZF1-AS1. QPCR and paired t-test were used to measure and compare expression levels of NOTCH-1 mRNA between two types of tissues (NSCLC vs. non-tumor) (a). The correlation between FEZF1-AS1 and NOTCH-1 mRNA was analyzed by linear regression (b). Data of 3 replicates were presented, ${ }^{*}, p<0.05$

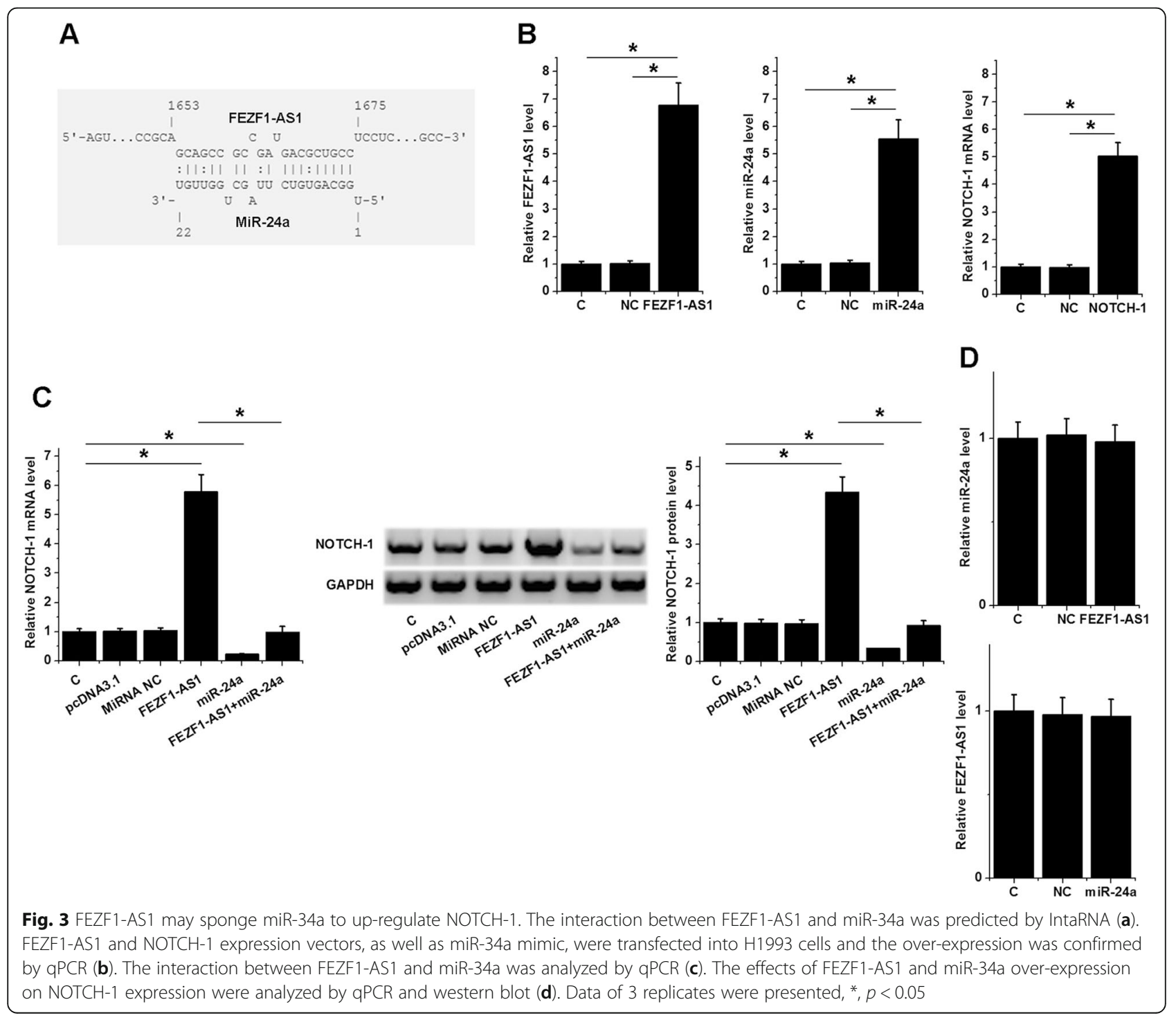


compared to non-tumor tissues (Fig. 1a, $p<0.05$ ). Expression levels of FEZF1-AS1 in NSCLC tissues among different clinical stages were compared. With the increase in clinical stages, significantly increased expression levels of FEZF1-AS1 were observed (Fig. 1b, $p<$ 0.05). Chi-squared test showed that expression levels of FEZF1-AS1 in NSCLC tissues were significantly correlated with patients' clinical stage and tumor grades, but not patients' gender, age, subtypes and drinking and smoking habits (Table 1).

\section{NOTCH-1 mRNA was up-regulated and positively} correlated with FEZF1-AS1

Analysis of RT-qPCR data revealed significantly higher expression levels of NOTCH-1 mRNA in NSCLC tissues compared to non-tumor tissues (Fig. 2a, $p<0.05$ ). Correlation analysis showed that, across NSCLC tissues, FEZF1-AS1 and NOTCH-1 were significantly and positively correlated (Fig. $2 \mathrm{~b}, p<0.05$ ).

\section{FEZF1-AS1 sponges miR-34a to up-regulate NOTCH-1}

The positive correlation between FEZF1-AS1 and NOTCH-1 mRNA indicated possible interaction between them, while miR-34a can target NOTCH-1. Therefore, the relationship between them was predicted. As shown in Fig. 3a, miR-34a can strongly bind to FEZF1-AS1. To further investigate the mechanism, FEZF1-AS1 or NOTCH-1 expression vector, or miR-34a mimic, was transfected into H1993 cells. QPCR showed that expression levels of miR34a, FEZF1-AS1 and NOTCH-1 were significantly upregulated compared to $\mathrm{C}$ and $\mathrm{NC}$ groups at $24 \mathrm{~h}$ posttransfection (Fig. 3b, $p<0.05$ ). Moreover, over-expression of miR-34a and FEZF1-AS1 did not significantly alter the expression of each other (Fig. 3c, $p>0.05$ ). In contrast, over-expression of FEZF1-AS1 resulted in up-regulated $\mathrm{NOTCH}-1$, while miR-34a over-expression mediated down-regulated $\mathrm{NOTCH}-1$ and the reduced effects of FEZF1-AS1 over-expression (Fig. 3d, $p<0.05$ ). Please check Supplementary Figure 1 for representative images of Western blot results.

FEZF1-AS1 promoted the invasion and migration of NSCLCs through the axis of NOTCH-1 and miR-34a Invasion (Fig. 4a) and migration (Fig. 4b) of H1993 cells in different transfection groups were analyzed by Transwell assay. Compared to $\mathrm{C}$ and $\mathrm{NC}$ groups, cells with NOTCH-1 or FEZF1-AS1 expression vector transfection exhibited significantly increased cell invasion and migration. MiR-34a played the opposite role and reduced the effects of FEZF1-AS1 over-expression $(p<0.05)$.

\section{Discussion}

We investigated the roles of FEZF1-AS1 in NSCLC. Our study revealed that FEZF1-AS1 was up-regulated in NSCLC. In addition, FEZF1-AS1 may sever as an endogenous sponge of miR-34a to up-regulate NOTCH-1 in NSCLC cells, thereby increasing the invasion and migration of cancer cells.

The function of FEZF1-AS1 has been investigated in many types of cancers. For instance, FEZF1-AS1 is up-regulated in colorectal cancer and can regulate PKM2 signaling to promote cancer cell metastasis and proliferation [14]. In multiple myeloma, FEZF1-AS1 is also overexpressed and over-expression of FEZF1-AS1 mediates the growth of tumor [16]. In a recent study, $\mathrm{He}$ et al. found that FEZF1-AS1 was over-expressed and is correlated with
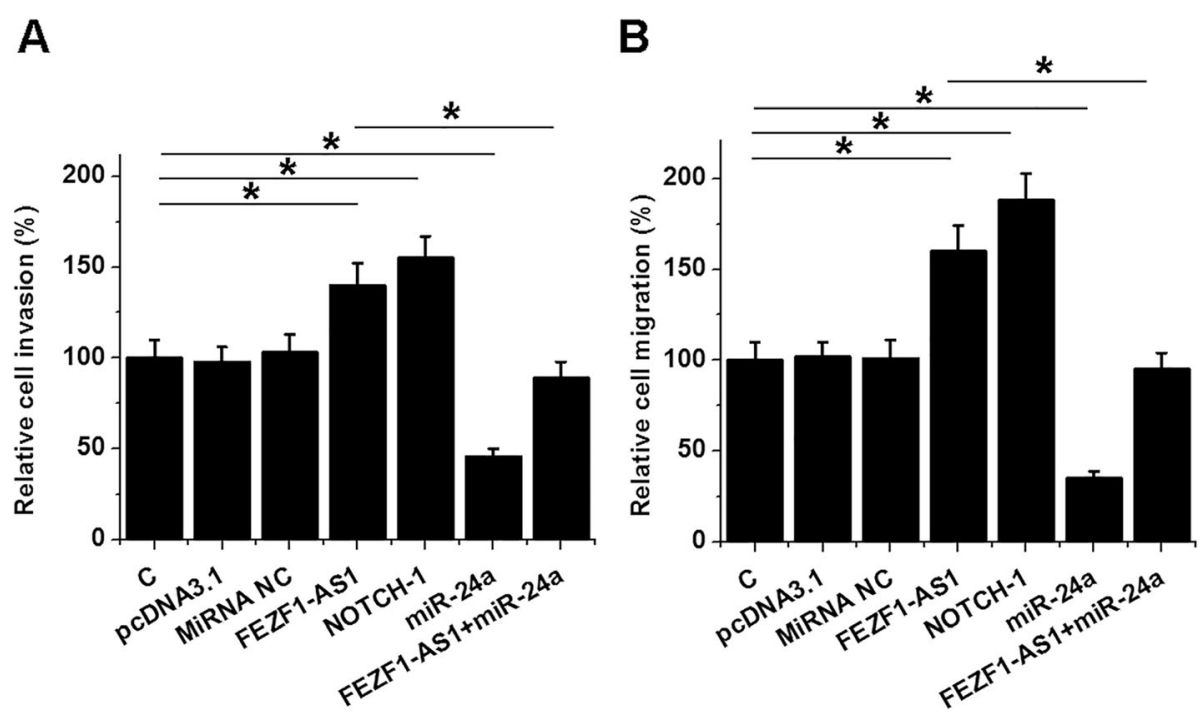

Fig. 4 FEZF1-AS1 promoted NSCLC cell invasion and migration through miR-34a and NOTCH-1. Transwell assays were performed to analyze the effects of transfections on the invasion (a) and migration (b) of H1993 cells. Data of 3 replicates were presented, ${ }^{*}, p<0.05$ 
epithelial-mesenchymal transition [15]. Consistently, this study also reported the up-regulation of FEZF1-AS1 in NSCLC, and the over-expression of FEZF1-AS1 resulted in the increased invasion and migration rates of NSCLC cells. Our study further the role of FEZF1-AS1 as an oncogenic IncRNA in NSCLC.

This study predicted the potential interactions between FEZF1-AS1 and miR-34a. It has been well established that miRNAs can regulate gene expression by direct cleavage or translation termination [17]. However, over-expression experiments showed that miR-34a has no regulatory roles in the expression of FEZF1-AS1. Therefore, miR-34a is not likely to target FEZF1-AS1. Recent studies have showed that IncRNAs may mimic the targets of miRNAs, thereby sponge miRNAs to attenuate their effects on silencing downstream genes. It is known that miR-34a can target $\mathrm{NOTCH}-1$ in glioblastoma to suppress cancer growth. In this study we also observed the downregulated NOTCH-1 at both mRNA and protein levels. Therefore, miR-34a may also target NOTCH1 in NSCLC. In addition, FEZF1-AS1 over-expression resulted in the up-regulated $\mathrm{NOTCH}-1$ at both mRNA and protein levels. Therefore, our data support the speculation that FEZF1-AS1 may sponge miR-34a to up-regulate $\mathrm{NOTCH}-1$, thereby promoting the invasion and migration of cancer cells. However, the mechanism remains to be further explored.

Our data may suggest FEZF1-AS1 as a potential therapeutic target for NSCLC. However, our study did not include clinical trials. Therefore, future studies are needed to perform clinical studies or animal model studies to further confirm our conclusions.

\section{Conclusion}

In conclusion, our study confirmed the oncogenic role of FEZF1-AS1 in NSCLC and we proposed that FEZF1AS1 may sponge miR-34a to up-regulate NOTCH-1 to promote cancer cell invasion and migration.

\section{Supplementary information}

Supplementary information accompanies this paper at https://doi.org/10. 1186/s12890-020-1154-6.

Additional file 1: Figure S1. Representative images of Western blot results. Original images here are of a biological replicate of Western blot. Data here is the same information as shown in Fig.4c.

\section{Abbreviation}

NSCLC: Non-small cell lung cancer; IncRNAs: Long (> $200 \mathrm{nt}$ ) non-coding RNAs; NOTCH-1: Notch homolog 1

Acknowledgments

Not applicable.

\section{Authors' contribution}

SXH conception, interpretation of data, revised the manuscript, supervision. $\mathrm{CJ}, \mathrm{JFH}, \mathrm{PHH}, \mathrm{DCM}, \mathrm{HLM}$ acquisition, analysis of data, software, drafted the manuscript. All the authors have approved the submitted version, and ensured the questions related to the accuracy or integrity of any part of the work.

Funding

Not applicable.

Availability of data and materials

The analyzed data sets generated during the study are available from the corresponding author on reasonable request.

Ethics approval and consent to participate

The present study was approved by the Ethics Committee of The Third Affiliated Hospital of Guangxi Medical University. The research has been carried out in accordance with the World Medical Association Declaration of Helsinki. All patients and healthy volunteers provided written informed consent prior to their inclusion within the study.

Consent for publication

Not applicable.

\section{Competing interests}

The authors declare that they have no competing interests.

\section{Author details}

1'Department of Radiotherapy, The Third Affiliated Hospital of Guangxi Medical University, No.13 Dancun Road, Nanning, Guangxi 530031, People's Republic of China. ${ }^{2}$ Department of Pathology, The Affiliated Tumor Hospital of Guangxi Medical University, Nanning, Guangxi 530031, People's Republic of China.

Received: 2 June 2019 Accepted: 20 April 2020

Published online: 29 April 2020

\section{References}

1. Bray F, Ferlay J, Soerjomataram I, et al. Global cancer statistics 2018: GLOBOCAN estimates of incidence and mortality worldwide for 36 cancers in 185 countries. CA Cancer J Clin. 2018;68(6):394-424.

2. Siegel RL, Miller KD, Jemal A. Cancer statistics, 2019. CA Cancer J Clin. 2019; 69(1):7-34.

3. Torre LA, Siegel RL, Jemal A. Lung cancer statistics $[M] / /$ lung cancer and personalized medicine. Cham: Springer; 2016. p. 1-19.

4. Jamal-Hanjani M, Wilson GA, McGranahan N, et al. Tracking the evolution of non-small-cell lung cancer. N Engl J Med. 2017;376(22):2109-21.

5. Chen W, Zheng R, Baade PD, et al. Cancer statistics in China, 2015. CA Cancer J Clin. 2016;66(2):115-32.

6. Jung KW, Won YJ, Oh CM, et al. Cancer statistics in Korea: incidence, mortality, survival, and prevalence in 2014. Cancer Res Treat. 2017;49(2):292305.

7. Islami F, Torre LA, Jemal A. Global trends of lung cancer mortality and smoking prevalence. Transl Lung Cancer Res. 2015;4(4):327-38.

8. Gazdar AF, Zhou C. Lung cancer in never-smokers: a different disease [M]// IASLC Thoracic Oncology. Cont Repository Only. 2018:23-9 e3.

9. Cooper WA. Molecular genetics of non-small cell lung cancer-current state of play. Pathology. 2016;48:S10.

10. Fender AW, Nutter JM, Fitzgerald TL, et al. Notch-1 promotes stemness and epithelial to mesenchymal transition in colorectal cancer. J Cell Biochem. 2015;116(11):2517-27.

11. Shao $S$, Zhao X, Zhang $X$, et al. Notch1 signaling regulates the epithelialmesenchymal transition and invasion of breast cancer in a slug-dependent manner. Mol Cancer. 2015;14(1):28.

12. Li Y, Guessous F, Zhang Y, et al. MicroRNA-34a inhibits glioblastoma growth by targeting multiple oncogenes. Cancer Res. 2009:69(19):7569-76.

13. Paraskevopoulou MD, Hatzigeorgiou AG. Analyzing miRNA-IncRNA interactions $[\mathrm{M}] / /$ long non-coding RNAs. New York: Humana Press; 2016. p. $271-86$. 
14. Bian Z, Zhang J, Li M, et al. LncRNA-FEZF1-AS1 promotes tumor proliferation and metastasis in colorectal Cancer by regulating PKM2 signaling. Clin Cancer Res. 2018;24(19):4808-19.

15. He R, Hu Zhang F, Shen N. LncRNA FEZF1-AS1 enhances epithelialmesenchymal transition (EMT) through suppressing E-cadherin and regulating WNT pathway in non-small cell lung cancer (NSCLC). Biomed Pharmacother. 2017;95:331-8.

16. Li Q, Chen $\mathrm{L}$, Hu N, et al. Long non-coding RNA FEZF1-AS1 promotes cell growth in multiple myeloma via miR-610/Akt3 axis. Biomed Pharmacother 2018;103:1727-32.

17. Carthew RW. Gene regulation by microRNAs. Curr Opin Genet Dev. 2006; 16(2):203-8

\section{Publisher's Note}

Springer Nature remains neutral with regard to jurisdictional claims in published maps and institutional affiliations.

Ready to submit your research? Choose BMC and benefit from:

- fast, convenient online submission

- thorough peer review by experienced researchers in your field

- rapid publication on acceptance

- support for research data, including large and complex data types

- gold Open Access which fosters wider collaboration and increased citations

- maximum visibility for your research: over $100 \mathrm{M}$ website views per year

At BMC, research is always in progress.

Learn more biomedcentral.com/submissions 